\title{
Auswirkungen der Klimaänderung auf die schweizerische Wasserkraftnutzung
}

\author{
R. Weingartner, B. Schädler, and P. Hänggi \\ Institute of Geography and Oeschger Centre for Climate Change Research, \\ University of Bern, Bern, Switzerland \\ Correspondence to: R. Weingartner (rolf.weingartner@giub.unibe.ch)
}

Received: 27 September 2012 - Revised: 18 March 2013 - Accepted: 25 March 2013 - Published: 16 December 2013

\begin{abstract}
Zusammenfassung. Hydraulic power contributes for a large part to electricity production in Switzerland. However, hydropower could be strongly affected by climate change. For that reason, the project CCWasserkraft - which results are summarised here - has been launched. In different studies, important aspects of the interactions between climate, hydrology and hydropower were investigated. Comprehensive simulations in representative catchments allowed quantifying the impacts of climate change on discharge and hydropower production. Finally, the results were extrapolated to whole Switzerland. The results show that significant changes are likely in the near future 2021-2050: an increase of hydropower production is projected for the winter half year, whereas stagnation or a decrease is expected in the summer half year. On the whole, the yearly hydropower production should remain constant or could even increase slightly. Projections for 2070-2099 remain uncertain. However, hydropower production from highly glaciated catchments located in southern and eastern Valais as well as catchments south of the Alps is expected to decline.
\end{abstract}

\section{Einleitung}

Der Wasserkreislauf ist direkt mit dem Klima verbunden, welches zunehmend auch anthropogenen Einflüssen unterworfen ist. Im Alpengebiet reagiert der Wasserkreislauf besonders sensitiv auf die Klimaänderung, weil ein veränderter saisonaler Auf- und Abbau der Schneedecke und das langfristige Abschmelzen der Gletscher einen unmittelbaren Einfluss auf die verfügbaren Wasserressourcen haben. Da in der Schweiz rund $56 \%$ der elektrischen Energie durch die Wasserkraft produziert wird (BFE, 2007), sind die klimabedingten Veränderungen des Wasserkreislaufes wichtig für die mittel- bis langfristige Planung der schweizerischen Stromversorgung wie auch für den Betrieb der einzelnen Wasserkraftanlagen. Das Bundesamt für Energie (BFE) hat in den letzten Jahren mehrere Studien zur Energieperspektive bzw. -strategie der Schweiz durchgeführt (BFE, 2011). Nebst der Beurteilung der Ausbau- und Neubaukapazitäten von Wasserkraftanlagen wurden auch die Auswirkungen der Klimaänderung in die Analysen miteinbezogen. In der Studie aus dem Jahr 2007 (BFE, 2007) ging man - im Vergleich zu heu- te - von einer klimabedingten Minderproduktion von $7 \%$ um 2035 und von $17 \%$ gegen Ende des 21. Jahrhunderts aus. Diese Aussagen basieren auf hydrologischen Simulationen in elf meist alpinen Einzugsgebieten (Horton et al., 2005). Ausgangspunkt bildeten dabei die Klimaszenarien des Projektes PRUDENCE (Christensen et al., 2002).

In den letzten Jahren haben sich die Voraussetzungen zur Abschätzung der klimabedingten hydrologischen Veränderungen wesentlich verbessert: Im europäischen Forschungsprojekt ENSEMBLES (Van Der Linden und Mitchell, 2009) wurden neue, umfassende regionale Klimaszenarien erarbeitet. Sie bildeten die Grundlage für verfeinerte Klimaszenarien für die Schweiz (CH2011, 2011; Bosshard, 2011; Bosshard et al., 2011). Auch in der hydrologischen Modellierung sind wesentliche Fortschritte erzielt worden (Viviroli et al., 2009; Magnusson et al., 2011; Hänggi, 2011).

Im Jahr 2008 lancierten swisselectric research (Forschungsstiftung der schweizerischen Stromverbundunternehmen Axpo, Alpiq und BKW) zusammen mit dem Bundesamt für Energie ein Forschungsprojekt, um zu einer neuen quantitativen Abschätzung der Auswirkungen der 


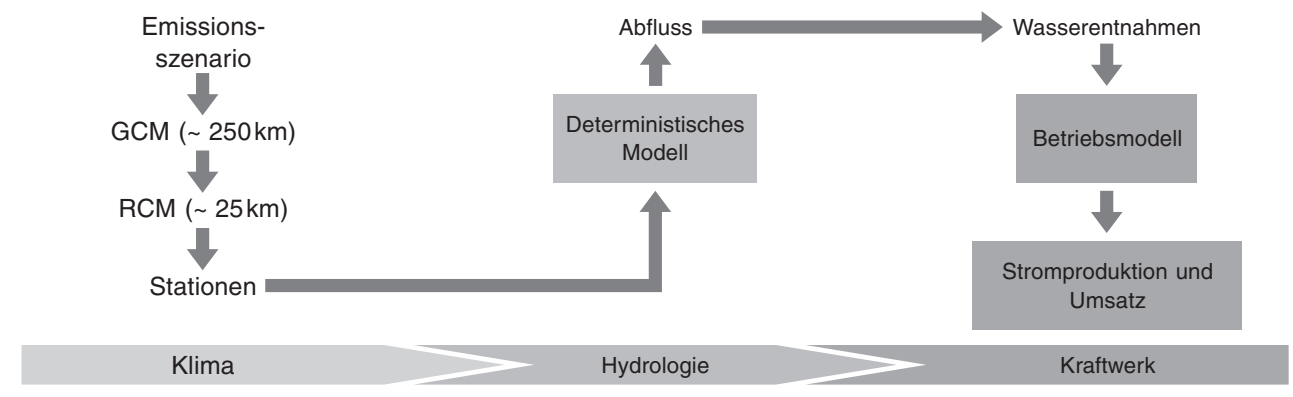

Abbildung 1. Schematische Darstellung der verwendeten Modellkette zur Analyse der Auswirkungen der Klimaänderung (aus Hänggi et al., 2011a).

Klimaänderung auf die Wasserressourcen und die Stromproduktion der Schweiz zu gelangen. Mitinitianten dieses im Folgenden als CCWasserkraft bezeichneten Projektes waren ferner die Dienststelle für Energie und Wasserkraft des Kantons Wallis und die Walliser Kraftwerksgesellschaft FMV SA. Die Zielsetzung des Projektes war es, die Auswirkungen der Klimaänderung zu untersuchen, und zwar

1. auf den Abfluss und insbesondere auf die Abflussregimes repräsentativer Einzugsgebiete der Schweiz,

2. auf den Betrieb und damit die Stromproduktion typischer Kraftwerksanlagen sowie

3. auf die Stromproduktion in der gesamten Schweiz.

Wichtige Rahmenbedingungen der Wasserkraftnutzung, wie Strompreis- oder Nachfrageveränderungen, wurden nicht berücksichtigt. Ziel des Projektes war es, den Effekt einer möglichen Klimaänderung isoliert zu quantifizieren. Am modular aufgebauten Forschungsprojekt waren insgesamt sechs Forschungsgruppen beteiligt. Es wurde vom Netzwerk Wasser im Berggebiet koordiniert und von Rolf Weingartner und Bruno Schädler vom Geographischen Institut der Universität Bern sowie Massimiliano Zappa von der Forschungsanstalt für Wald, Schnee und Landschaft geleitet.

Im vorliegenden Aufsatz sollen die wichtigsten Ergebnisse von CCWasserkraft in knapper Form dargestellt werden. Der Projekt-Synthesebericht (SGHL und CHy, 2011), Fachberichte (Fachberichte, 2011) und Publikationen in internationalen Fachzeitschriften zu spezifischen Aspekten vermitteln umfassende Einblicke in die Ergebnisse. Der vorliegende Bericht stellt die wichtigsten Ergebnisse im Sinne eines Review-Artikels zusammen.

\section{Methoden}

Der Abfluss bildet die Schlüsselgrösse des Projekts CCWasserkraft. Es geht einerseits darum, die Entwicklung der Abflussverhältnisse im Zeitraum 1900 bis $2100 \mathrm{zu}$ verstehen und andererseits die Auswirkungen dieser Veränderungen auf die Stromproduktion zu simulieren. Dazu gelang- ten drei miteinander verbundene Modellsysteme zur Anwendung (Abb. 1). In einzelnen Teilprojekten wurden alle drei Modellsysteme gekoppelt (Hänggi et al., 2011a; Stähli et al., 2011), in anderen Teilprojekten standen hydrologische bzw. hydro-glaziologische Modelle in Kombination mit der Klimamodellierung im Mittelpunkt (Farinotti et al., 2011; Hänggi, 2011).

Die verschiedenen Klimamodellketten des EU-Projektes ENSEMBLES (Van der Linden und Mitchell, 2009), welche durch das A1B Emissionsszenario (vgl. IPCC, 2007) angetrieben werden, bilden den Kern der Klimamodellierung. In der Gesamtheit der Emissionsszenarien nach IPCC (2007) stellt A1B ein mittleres Szenario dar, weshalb es im Projekt CCWasserkraft hauptsächlich als Basis diente. Die Klimamodellketten bestehen aus globalen Klimamodellen (GCMs) mit grober Auflösung, deren Ergebnisse mittels regionaler Klimamodelle (RCMs) dynamisch auf eine Auflösung von $25 \mathrm{~km}$ herunterskaliert wurden. Dies ist notwendig, damit die Prozesse in der Atmosphäre über dem komplexen Gelände der Alpen überhaupt modelliert werden können. Für die Verwendung in kleinräumigen hydrologischen Untersuchungen war eine Aufbereitung notwendig. Wie in BAFU $(2012,25)$ beschrieben, wurden die Ergebnisse der regionalen Klimamodelle ,mittels inverser Distanzgewichtung der nächstliegenden 4 Gitterpunkte auf einzelne Standorte (189 Temperatur- und 565 Niederschlagsstandorte) interpoliert. [...] Zudem musste das eigentliche Klimasignal von der natürlichen Variabilität getrennt werden. Dies erfolgte durch eine spektrale Filterung der Jahresgänge und deren anschliessender Anwendung in der Delta-Change-Methode (Bosshard et al., 2011)“: Für jeden Standort und jeden Tag im Jahr wurde ein Klimaänderungssignal der Temperatur und des Niederschlags berechnet, welches sich aus einem Vergleich zwischen den modellierten Werten der Szenarioperiode SCE (2021-2050) und jenen der Kontrollperiode CTL (1980-2009) ergibt.

Da insgesamt zehn Kombinationen verschiedener GCMs und RCMs berücksichtigt wurden, liegen nun zehn unterschiedliche klimatische Modellantriebe für die hydrologischen und hydro-glaziologischen Modelle vor (Modellkette Klima-Hydrologie (K-H); siehe Untersuchungsgebiete in 


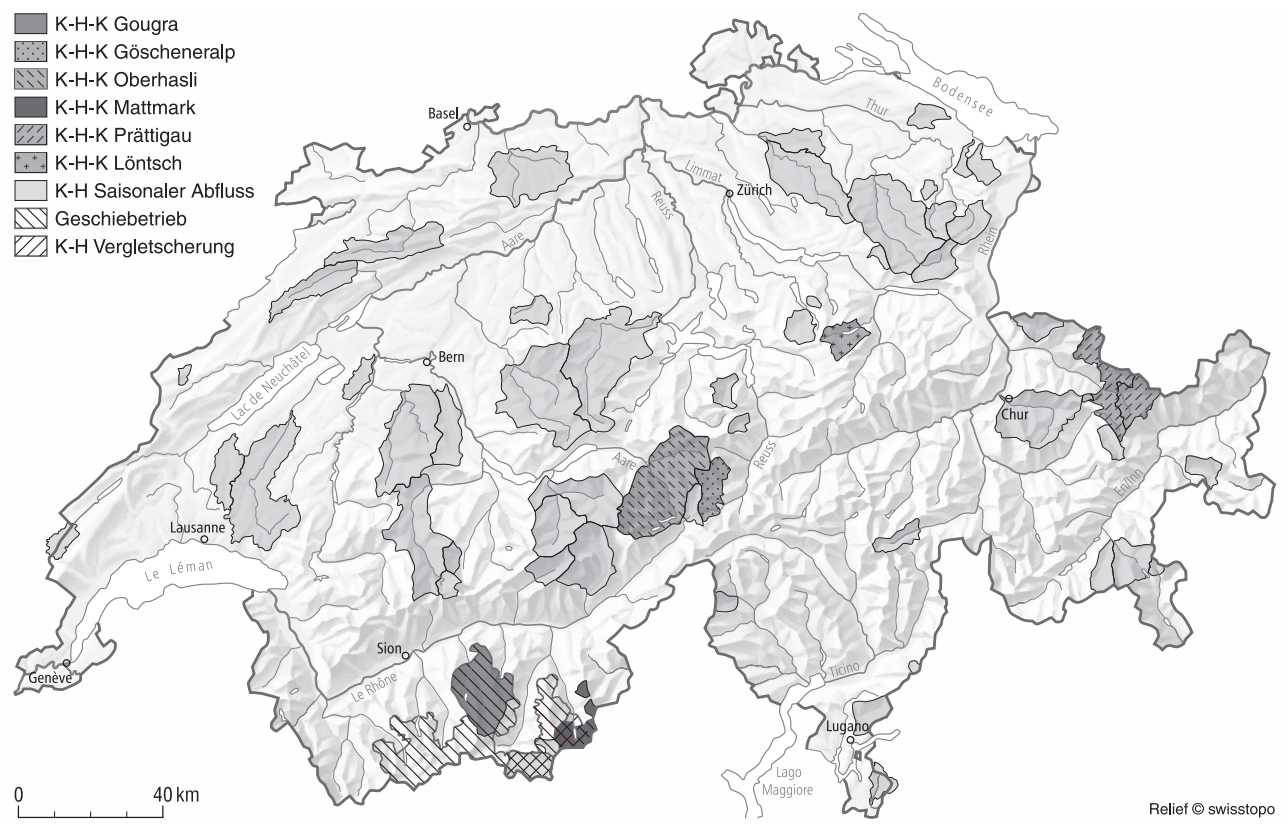

Abbildung 2. Untersuchungsgebiete des Projektes CCWasserkraft mit thematischem Fokus. Es wurden Untersuchungen zu Veränderungen innerhalb der Modellkette Klima-Hydrologie (K-H; Analyse des saisonalen Abflusses und/oder der Massenbilanz von Gletschern), KlimaHydrologie-Kraftwerk (K-H-K; zusätzlich Analyse der Betriebsweise von Wasserkraftwerken) und zum Geschiebetrieb durchgeführt.

Abb. 2). Es gelangten verschiedene deterministische hydrologische Modelle zum Einsatz: PREVAH (Viviroli et al., 2009), BERNHYDRO (Hänggi, 2011) und GERM (Huss et al., 2008). Der Vergleich mit beobachteten Abflüssen sowie die berechneten Gütemasse zeigten, dass die Modelle die hydrologischen Bedingungen in den untersuchten Repräsentativgebieten gut wiedergeben können. Bei der Wahl der Repräsentativgebieten waren mehrere Kriterien ausschlaggebend: (1) lange natürliche bzw. anthropogen nicht beeinflusste Messreihen, (2) Abdeckung der 16 Abflussregimetypen der Schweiz (vgl. Weingartner und Aschwanden, 1992), (3) glaziologisch gut dokumentierte Gebiete und (4) Gebiete mit einer für die Wasserkraftnutzung typischen Anlage (s. Balmer, 2011). Kriterium 1 kam bei der Analyse von Hänggi und Weingartner (2011) zum Tragen; hier ging es darum, die zeitliche Entwicklung von Niederschlag und Abfluss seit 1800 am Beispiel des Rheins in Basel zu untersuchen. Über repräsentative schweizerische Einzugsgebiete mit langen natürlichen Messreihen (Kriterium 1 und 2) untersuchten Hänggi et al. (2011b) die Veränderung der Abflussregimes zwischen 1900 und 2050. Farinotti et al. (2011) simulierten das Abflussverhalten stark vergletscherter Einzugsgebiete im selben Zeitraum (Kriterium 3). Gletscher sind von der Klimaänderung markant betroffen.

Schliesslich wurde die ganze Modellkette der Abb. 1 in Gebieten, die dem Kriterium 4 genügen, eingesetzt (Hänggi et al., 2011a; Stähli et al., 2011), so dass direkte Aussagen zu den Auswirkungen der Klimaänderung auf die Stromproduktion gemacht werden können. Bei dieser Kraftwerks- modellierung wurden die aus den verschiedenen Modellantrieben resultierenden Abflussganglinien in ein Betriebsmodell der untersuchten Kraftwerksanlagen eingegeben, so dass die Auswirkungen auf die Stromproduktion und den Umsatz berechnet werden konnten (Modellkette Klima-HydrologieKraftwerk (K-H-K); siehe Untersuchungsgebiete in Abb. 2). Unter anderem gelangten die Software TimeSteps-Energy $2010^{\circledR}$ (Blöchlinger et al., 2004) und WABES (Af-Colenco AG, 2004) zum Einsatz. Sie berechnen unter Berücksichtigung der simulierten Abflüsse bzw. Zuflüsse den optimalen Einsatz der Kraftwerksanlagen zur Ertragsmaximierung. Wichtige Rahmenbedingungen wie Strompreis und Nachfragemuster wurden von den heutigen Bedingungen auf die Szenarioperiode 2021-2050 übertragen und für jede Berechnung unverändert belassen: Die Bildung in-sich konsistenter Szenarien unter Berücksichtigung aller Rahmenbedingungen ist äusserst komplex und konnte mit den gegebenen Ressourcen nicht durchgeführt werden. Es wurde somit versucht, den Effekt einer möglichen Klimaänderung isoliert zu quantifizieren.

Abbildung 2 vermittelt eine Übersicht über die untersuchten Gebiete mit ihrer zentralen Fragestellung. Da der Grossteil der Wasserkraft im Alpenraum produziert wird, liegen die integralen, die ganze Modellkette abdeckenden Fallstudien vor allem im Alpenraum (Gebiete K-H-K). Zusätzlich - und hier nicht weiter beschrieben - wurden auch die Auswirkungen veränderter Abflussverhältnisse auf den Geschiebetransport untersucht (Raymond-Pralong et al., 2011). 


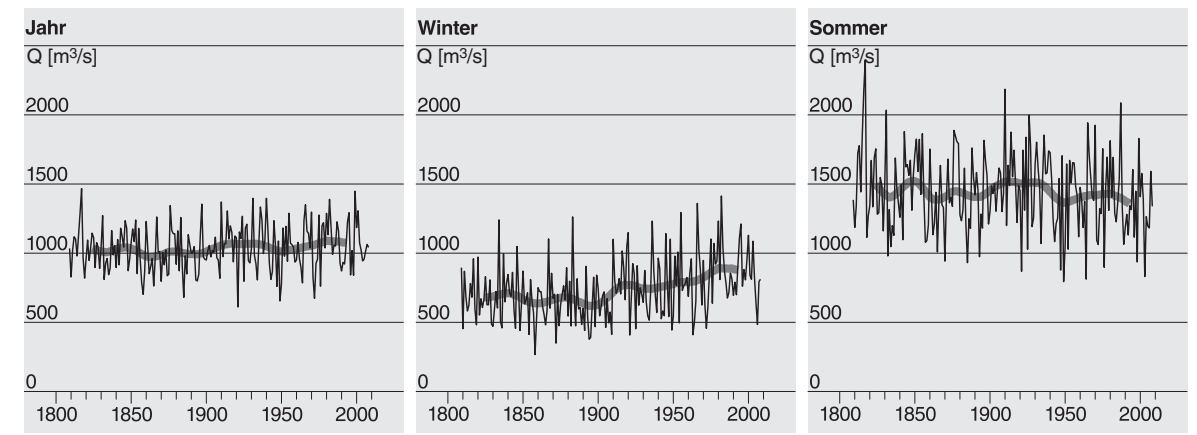

Abbildung 3. Mittlere Jahres- (Oktober bis September), Winter- (Dezember bis Februar) und Sommerabflussmengen (Juni bis August) des Rheins in Basel 1808-2007: Einzeljahre und 30-jährige gleitende Mittelwerte.

Ein wichtiges Ziel des hier vorgestellten Projektes war es, die in den Untersuchungsgebieten (Abb. 2) erzielten Ergebnisse zu nutzen, um letztlich für die gesamte Schweiz abzuschätzen, wie sich die Stromproduktion im Zeitraum 2021-2050 gegenüber heute verändern wird. Dazu wurde eine Hochrechnung durchgeführt (Hänggi et al., 2011c). Sie basiert auf einer Kombination zweier Klassierungen: Bei der ersten Klassierung wurden Wasserkraftanlagen, die aus technischer und naturräumlicher Sicht ähnlich sind, in Gruppen zusammengefasst (Balmer, 2011). Bei der zweiten Klassierung wurden mit statistischen Methoden Regionen mit einem ähnlichen Klimaänderungssignal identifiziert. Bei der kombinierten Klassierung ergaben sich schliesslich 24 Kraftwerksgruppen, die sich bezüglich der technischnaturräumlichen Eigenschaften und/oder des zu erwartenden Klimaänderungssignals unterscheiden. Diesen Kraftwerksgruppen wurden in einem nächsten Schritt die Resultate der Fallstudien, d.h. die in den beiden Fallstudien festgestellten zukünftigen Veränderungen von Zuflussmenge und Stromproduktion, zugeordnet. Da für jede Kraftwerksgruppe bekannt ist, wie gross ihr Beitrag zur gesamten Stromproduktion der Schweiz ist (Balmer, 2011), konnte mit Hilfe der genannten Zuordnung eine Hochrechnung auf die ganze Schweiz durchgeführt werden.

\section{Ergebnisse und Diskussion}

Das Projekt CCWasserkraft hat eine Fülle von Ergebnissen erzeugt, welche weit über den Rahmen dieses Artikels hinausgehen. Im Folgenden sollen gemäss den in der Einleitung genannten Zielsetzungen vier massgebliche Aspekte hervorgehoben werden:

\subsection{Projizierte Klimaänderung}

In der Schweiz kann, neben einem Anstieg der Temperatur in den vergangenen $30 \mathrm{Jahren}$ um $0.35^{\circ} \mathrm{C}$ pro Jahrzehnt, für das 20. Jahrhundert eine Zunahme der Jahresniederschlagsmenge um ca. $8 \%$ festgestellt werden (Me-
teoSchweiz, 2012). Die in CCWasserkraft erarbeiteten und verwendeten Klimaszenarien gehen von einem weiteren Anstieg der mittleren Temperaturen in allen Jahreszeiten zwischen 1.0 und $1.5^{\circ} \mathrm{C}$ bis $2021-2050$ gegenüber 1980-2009 aus (Bosshard, 2011). Dieser Anstieg ist schweizweit relativ homogen und liegt deutlich höher als die in der Vergangenheit gemessene natürliche Variabilität. Für den Niederschlag 2021-2050 kann auf der Alpennordseite ein positives Änderungssignal gegenüber 1980-2009 festgestellt werden, welcher in der Nordostschweiz am deutlichsten sichtbar ist. Auf der Alpensüdseite projizieren die Modelle hingegen eine leichte Abnahme der Jahresniederschlagsmengen. Bis 20702099 akzentuieren sich die Projektionen weiter, womit die Änderungen deutlicher hervortreten.

\subsection{Auswirkungen der Klimaänderung auf den Abfluss und insbesondere die Abflussregimes}

Für den Rhein in Basel liegt seit 1808 eine homogene Abflussmessreihe vor (Abb. 3). Das Einzugsgebiet mit einer Fläche von rund $36000 \mathrm{~km}^{2}$ entwässert einen grossen Teil der nordalpinen Schweiz. Die Abflussmessreihe ermöglicht deshalb eine zeitlich umfassende, räumlich integrale Analyse zur Veränderung der hydrologischen Verhältnisse auf der Alpennordseite. Statistische Untersuchungen der zeitlichen Veränderung der Wahrscheinlichkeitsdichtefunktionen von Lufttemperatur, Niederschlag und Abfluss erlauben Aussagen zur Veränderung der jährlichen und saisonalen Mittel sowie der interannuellen Variabilität. Die folgenden Punkte halten die wichtigsten Ergebnisse der Studie von Hänggi und Weingartner (2011) fest:

- Im Winter (Dezember bis Februar) hat der mittlere Abfluss signifikant zugenommen (vgl. Abb. 3): Im Wesentlichen hat der kombinierte Effekt von drei Faktoren zu dieser Abflusszunahme geführt: (1) Zunahme der Niederschlagsmengen, (2) Zunahme der Lufttemperatur und damit Erhöhung des direkt abflusswirksamen flüssigen Niederschlags und (3) Zunahme der Zuflussmengen aus den Stauseen, deren Speicherkapazität 

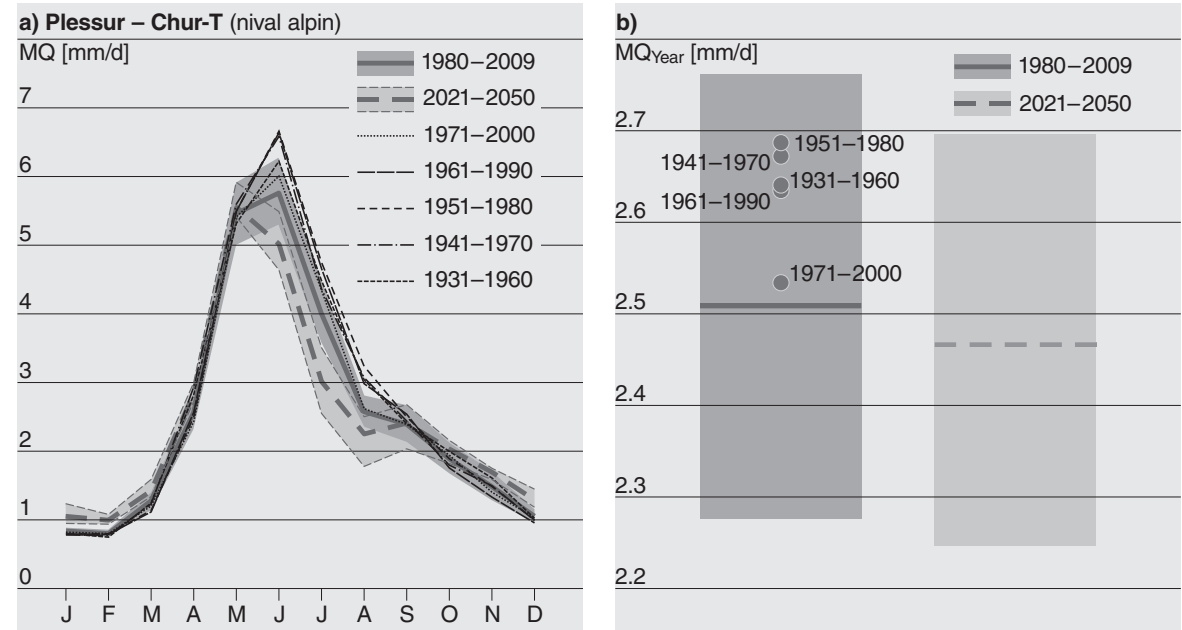

Abbildung 4. Veränderung des Abflussregimes (a) und der mittleren Abflusshöhen (b) der Plessur-Chur (aus Hänggi et al., 2011b). Linien (Punkte in b): Mittlere Regimes (mittlere Abflusshöhen) 1931-1960 bis 1980-2009, inkl. Abschätzung für 2021-2050. Die Bandbreite der monatlichen (jährlichen) Abflusshöhen ist zusätzlich für 1980-2009 und 2021-2050 angegeben.

im 20. Jahrhundert stark ausgebaut wurde. Die Jahr-zuJahr-Variabilität liegt heute in einer ähnlichen Grössenordnung wie zu Beginn des 19. Jahrhunderts; sie erreichte ein Maximum um die Mitte des 20. Jahrhunderts.

- Auch im Frühling (März bis Mai) hat der mittlere Abfluss wie auch dessen interannuelle Variabilität signifikant zugenommen. Entscheidende Einflussfaktoren sind hier wiederum die höheren Niederschlagsmengen und die räumlich-zeitlichen Veränderungen der Schneedecke. Letztere bedeuten ein temperaturbedingter Anstieg der Schneefallgrenze und ein früheres Eintreten der Schmelze.

- Im Sommer (Juni bis August; vgl. Abb. 3) und Herbst (September bis November) haben sich die mittleren Abflüsse, aber auch der Niederschlag nicht signifikant verändert.

- Beim mittleren Jahresabfluss (Oktober bis September) resultiert daraus eine zwar insgesamt eher kleine, aber statistisch noch signifikante Zunahme sowohl der Abflussmengen als auch der Jahr-zu-Jahr Variabilität (vgl. Abb. 3).

Insgesamt zeigen die Ergebnisse, dass sich die hydrologischen Rahmenbedingungen für die Wasserkraftproduktion im Laufe der Zeit stetig verbessert haben. So führt beispielsweise die Abflusszunahme im Winter direkt zu einer Erhöhung der Stromproduktion von Laufkraftwerken (vgl. Kapitel 3.4).

Im Folgenden werden die Veränderung der Abflüsse auf der Basis der für die schweizerischen Abflussregimes repräsentativen mesoskaligen Einzugsgebiete auf einer Zeitskala
1951 bis 2100 analysiert. Die Ergebnisse in Tabelle 1 zeigen, dass die Veränderungen des Abflussregimes vor allem im Alpenraum ausgeprägt sind: Das Abflussregime der untersuchten Repräsentativgebiete hat sich und/oder wird sich um eine bis zwei Regime-Stufen verändern (vgl. Lesebeispiel in Tabelle 1). Grund dafür sind Veränderungen in den Prozessen der Eis- und Schneeschmelze, wie anhand der nachfolgenden zwei Beispiele gezeigt wird:

Die Plessur (Messstelle Chur) ist eine Vertreterin des nivalen, also von der Schneeschmelze gesteuerten Regimes (vgl. Tabelle 1 und Abb. 4). Typisch für ein nivales Regime ist die folgende Rangfolge der grössten mittleren Monatsabflüsse: Juni-Mai-Juli-August (Weingartner und Aschwanden, 1992). Im Zukunftsszenario 2021-2050 ändert sich diese Abfolge, da die Abflüsse im Juni bis August infolge der früher einsetzenden Schneeschmelze markant abnehmen werden. Dadurch werden die mittleren Abflüsse im Mai und April relativ gesehen bedeutender. Die Abflüsse im Winter werden zunehmen. Insgesamt wandelt sich das Regime zum Typ nival de transition (vgl. Tabelle 1). Allerdings verändern sich dabei die Jahresabflussmengen nicht signifikant; d.h., es findet insgesamt „nur“ eine saisonale Verschiebung im Abflussverhalten statt.

Saisonale Verschiebungen sind auch beim Abflussregime der von der Gletscherschmelze geprägten Simme zu beobachten (vgl. Tabelle 1 und Abb. 5). Massgeblich hierfür ist das durch die negative Massenbilanz der Gletscher bedingte höhere Angebot an Schmelzwasser. Dadurch haben und werden sich sowohl das saisonale Abflussmuster als auch die Jahresabflussvolumina verändern. Der Zeitpunkt des Maximums an Schmelzwasserzufluss aus dem Reserveabbau der schwindenden Gletscher ist abhängig von der Gletschergrösse, und variiert somit von Gebiet zu Gebiet. Farinotti et 
Tabelle 1. Abschätzung der zeitlichen Veränderungen der Abflussregimes (Klassifikation nach Weingartner und Aschwanden, 1992) in repräsentativen Einzugsgebieten der Schweiz mit Hilfe von Klima- und hydrologischen Modellen (aus SGHL und CHy, 2011; Emissionsszenario A1B, Bosshard et al., 2011). Markiert sind die Einzugsgebiete mit Regimeänderungen. Lesebeispiel: Für das Einzugsgebiet Simme Oberried liegen Daten zur Periode 1951-1980 (H) und 1980-2009 (K) vor. Zudem wurden die Veränderungen des Abflusses für die Szenarioperiode 2021-2050 (S1) abgeschätzt. Für Szenarioperiode 2070-2099 (S2) liegen in diesem Falle keine Abschätzungen vor. Das heute (K) von der Gletscherschmelze gesteuerte Regime b-glaciaire wird sich in Zukunft (S1) zu einem a-glacio-nivalen Regime wandeln mit einer entsprechenden Abnahme der Bedeutung der Eisschmelze.

\begin{tabular}{|c|c|c|c|c|c|c|c|c|c|c|c|c|c|c|c|c|c|c|c|c|c|c|c|c|}
\hline Abflussregimetypen & & 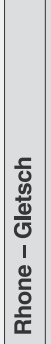 & 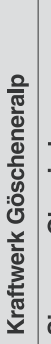 & 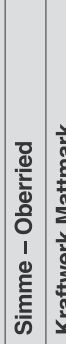 & 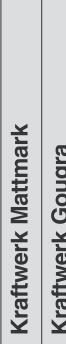 & 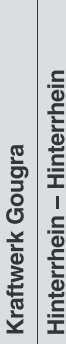 & 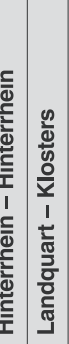 & 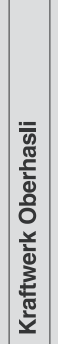 & 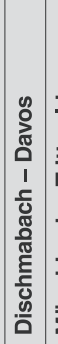 & 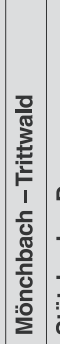 & 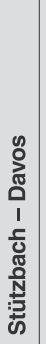 & 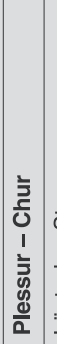 & 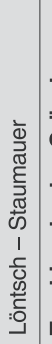 & 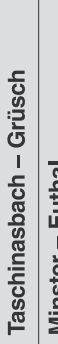 & 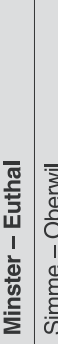 & 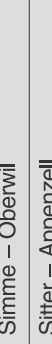 & 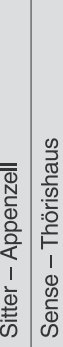 & 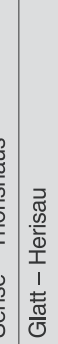 & 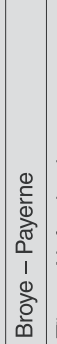 & 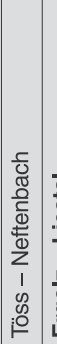 & 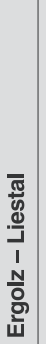 & 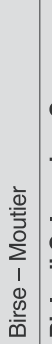 & 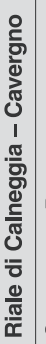 & 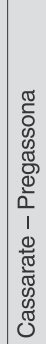 \\
\hline a-glaciaire & 舟 & H K & $k$ & & & & & & & & & & & & & & & & & & & & & \\
\hline b-glaciaire & A & s1 & & H K & k & & & & & & & & & & & & & & & & & & & \\
\hline a-glacio-nival & A & & & s1 & & \begin{tabular}{l|l}
$K$ & $H$ \\
\end{tabular} & H & & & & & & & & & & & & & & & & & \\
\hline b-glacio-nival & $A$ & & s1 & & s1 & k & K H K & $K$ & H & & & & & & & & & & & & & & & \\
\hline nivo-glaciaire & A & & & & & \begin{tabular}{l|l} 
s1 & s1
\end{tabular} & $\$ 1$ & s1 & K & K & K & & & & & & & & & & & & & \\
\hline nival alpin & 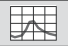 & & S2 & & \$2 & & & s2 & s1 & s1: & s1 & н К & $\mathbf{k}$ & H & & & & & & & & & & \\
\hline nival de transition & $N$ & & & & & s2 & & & & & & s1 & & \begin{tabular}{c|c}
$K$ & 1 \\
$S 1$ & 1 \\
\end{tabular} & $\begin{array}{ll}\mathrm{H} & \mathrm{H} \\
\mathrm{S}\end{array}$ & $\begin{array}{lll}4 K & H \\
S 1 & & \\
\end{array}$ & $\begin{array}{l}H K \\
\text { S1 }\end{array}$ & & & & & & & \\
\hline nivo-pluvial préalpin & 基 & & & & & & & & & & & & & & $\begin{array}{l}\mathbf{K} \\
\mathbf{S 1}\end{array}$ & & $\begin{array}{l}\mathrm{HK} \\
\mathrm{S1}\end{array}$ & $\begin{array}{l} \\
\end{array}$ & & & & & & \\
\hline pluvial supérieur & 册 & & & & & & & & & & & & & & & & & H & & & & & & \\
\hline pluvial inférieur & 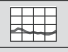 & & & & & & & & & & & & & & & & & & H H & H K & & & & \\
\hline nivo-pluvial jurassien & 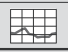 & & & & & & & & & & & & & & & & & & & & $\kappa$ & H & & \\
\hline pluvial jurassien & 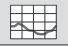 & & & & & & & & & & & & & & & & & & & & H & & & \\
\hline nival méridional & A & & \multirow{4}{*}{\multicolumn{8}{|c|}{$\begin{array}{l}\text { H HADES (1951-1980) } \\
\text { K Kontrollperiode }(1980-2009) \\
\text { S1 Szenario } 1(2021-2050) \\
\text { S2 Szenario } 2(2070-2099)\end{array}$}} & & & & & & & & & & & & & H K & \\
\hline nivo-pluvial méridional & 且 & & & & & & & & & & & & & & & & & & & & & & s1 & \\
\hline pluvio-nival méridional & 姃 & & & & & & & & & & & & & & & & & & & & & & & $\begin{array}{l}\mathrm{H} \mathrm{K} \\
\mathrm{S1}\end{array}$ \\
\hline pluvial méridional & 姃 & & & & & & & & & & & & & & & & & & & & & & & \\
\hline
\end{tabular}

Tabelle 2. Mittlere beobachtete Veränderungen der mittleren jährlichen und saisonalen Abflüsse in den repräsentativen Einzugsgebieten im Zeitraum 2021-2050 gegenüber 1980-2009 (nach Hänggi et al., 2011b).

\begin{tabular}{lccc}
\hline Regimes & $\begin{array}{c}\text { Winter } \\
\text { (Oktober bis März) }\end{array}$ & $\begin{array}{c}\text { Sommer } \\
\text { (April bis September) }\end{array}$ & $\begin{array}{c}\text { Jahr } \\
\text { (Oktober bis September) }\end{array}$ \\
\hline Glaziale Regimes & +20 bis $+30 \%$ & +1 bis $+15 \%$ & +5 bis $+15 \%$ \\
Nivale Regimes & +6 bis $+17 \%$ & -15 bis $-4 \%$ & -3 bis $+4 \%$ \\
Pluviale Regimes Alpennordseite & +2 bis $+5 \%$ & -8 bis $-2 \%$ & 0 bis $+5 \%$ \\
Südalpine Regimes & -5 bis $+16 \%$ & -11 bis $-2 \%$ & -4 bis $+6 \%$ \\
\hline
\end{tabular}

al. (2011) konnten anhand von Untersuchungen in stark vergletscherten Einzugsgebieten zeigen, dass gegenwärtig die Jahresabflussvolumina am höchsten sind und dass diese ab Mitte des 21. Jh. wieder auf das Niveau der ersten Hälfte des 20. Jh. zurückgehen werden. Ende des 21. Jh. wird das Abflussverhalten dann aber nicht mehr von der Gletscherschmelze, sondern von der jahreszeitlichen Verteilung der Schneeschmelze und vom Niederschlag geprägt sein.
Tabelle 2 fasst die Ergebnisse der Modellierungen in den sechzehn repräsentativen Einzugsgebieten zusammen. Die grössten Änderungen sind in den heute noch stark vergletscherten Einzugsgebieten zu beobachten: Hier werden die Abflüsse sowohl auf Jahres- wie auch Halbjahresbasis signifikant zunehmen. Bei den restlichen nordalpinen Einzugsgebieten werden die Jahresvolumina auf einem ähnlichen Niveau verharren, während die Abflüsse im Winter 

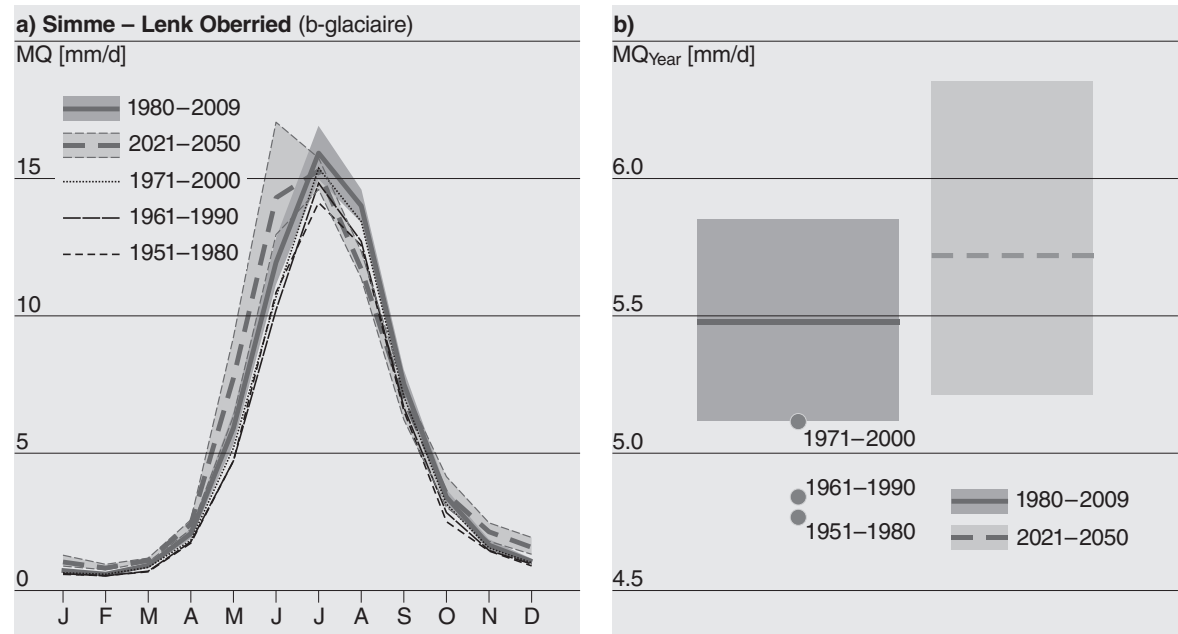

Abbildung 5. Wie Abb. 4, aber für Simme-Oberried (aus Hänggi et al., 2011b).
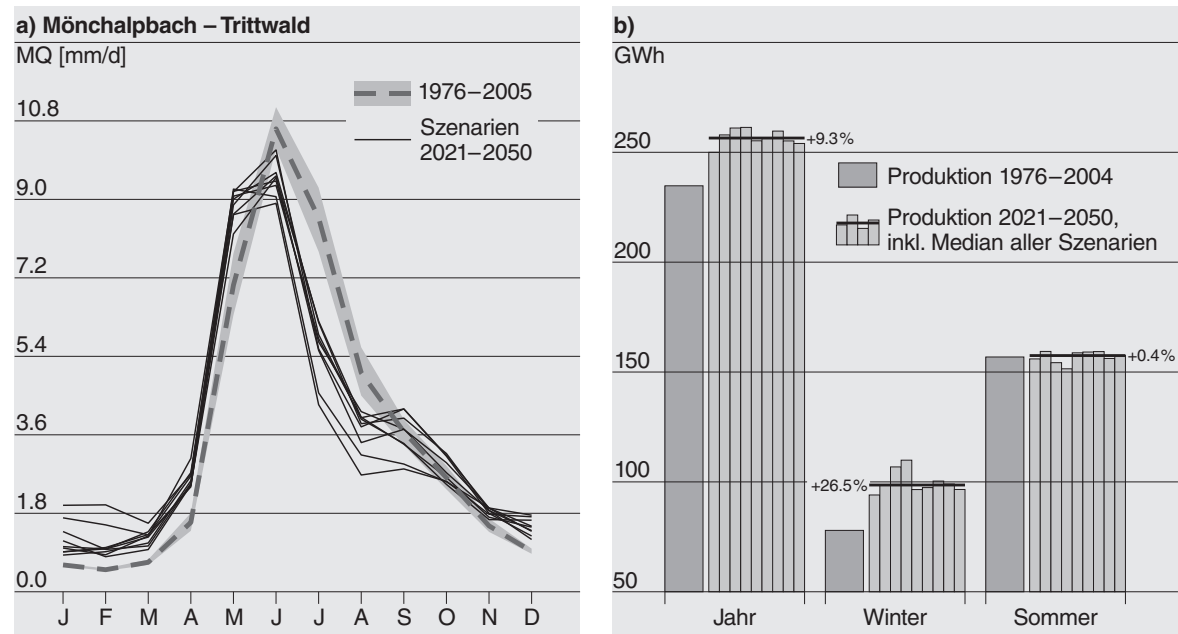

Abbildung 6. Beispiel zur Veränderung der Abflussregimes im Zuflussgebiet des Kraftwerks Prättigau und Auswirkungen der Klimaänderung auf die Stromproduktion (nach Hänggi et al., 2011a).

überall zunehmen und im Sommer überall abnehmen werden. Schlecht mit Daten belegt sind die Ergebnisse auf der Alpensüdseite: Unter Berücksichtigung der Ergebnisse der Klimamodellierung (Bosshard et al., 2011) ist dort vor allem in tieferen Lagen mit einem Rückgang der Abflüsse zu rechnen.

\subsection{Auswirkungen der Klimaänderung auf den Betrieb und damit die Stromproduktion typischer Kraftwerksanlagen}

Abflussmenge, Stromproduktion und Umsatz sind nicht immer linear miteinander verbunden. Entscheidend für den $\mathrm{Zu}$ sammenhang dieser Faktoren sind der Kraftwerkstyp und die individuelle Auslegung der einzelnen Anlagen. Im Rahmen des hier vorgestellten Projekts CCWasserkraft wurden des- halb verschiedene Fallstudien durchgeführt, bei denen die ganze Modellkette (Abb. 1) analysiert wurde, um so zu Aussagen über die Auswirkungen der Klimaänderung auf die Stromproduktion zu gelangen. Im Folgenden soll beispielhaft die „Fallstudie Kraftwerke Prättigau“ diskutiert werden (Hänggi et al., 2011a). Die Kraftwerke Prättigau repräsentieren einen Anlagetyp, dessen zugehörige Kraftwerke insgesamt rund $9 \%$ der schweizerischen Hydroelektrizität erzeugen (Hänggi et al., 2011c).

Die KW Prättigau liegen im Kanton Graubünden; sie umfassen neun Wasserfassungen, drei Kraftwerksstufen und kleine Saisonspeicher. Die Simulationen, welche auf acht verschiedenen Realisationen des A1B-Emissionsszenarios und auch einer Realisation des A2-Emissionsszenarios beruhen, zeigen, dass sich die heute meist glazio-nivalen 
Tabelle 3. Relative Veränderung der mittleren Stromproduktion aus Wasserkraft in der Periode 2021-2050 im Vergleich zur Periode 19802009 (aus Hänggi et al., 2011c).

\begin{tabular}{lccc}
\hline Variante & $\begin{array}{c}\text { Winter } \\
\text { (Oktober bis März) }\end{array}$ & $\begin{array}{c}\text { Sommer } \\
\text { (April bis September) }\end{array}$ & $\begin{array}{c}\text { Jahr } \\
\text { (Oktober bis September) }\end{array}$ \\
\hline Optimistisch & $+10.1 \%$ & $-4.4 \%$ & $+1.9 \%(0.7 \mathrm{TWh})$ \\
Pessimistisch & $+10.1 \%$ & $-6.3 \%$ & $+0.9 \%(0.3 \mathrm{TWh})$ \\
\hline
\end{tabular}

Abflussregimes (vgl. Tabelle 1) in den sieben Teileinzugsgebieten bis zur nahen Zukunft (Periode 2021-2050) deutlich verändern werden (Abb. 6): Es ist mit höheren Abflüssen von September bis Mai und mit tieferen Abflüssen von Juni bis August zu rechnen. Auf die Jahresabflussvolumina wirken sich diese saisonalen Veränderungen allerdings nur leicht aus; es werden Zunahmen von 1 bis $8 \%$ erwartet. Die Veränderungen des Abflussregimes haben nun aber weitreichende Auswirkungen auf die Stromproduktion. Wie Abb. 6 zeigt, haben sie eine winterliche Produktionssteigerung um 20 bis $40 \%$ zur Folge - bei einer kaum veränderten Sommerproduktion. Über das ganze Jahr gesehen ergibt dies eine Zunahme um rund 9\%. Die Produktionssteigerung im Winter ist darauf zurückzuführen, dass der zusätzlich anfallende Abfluss vollständig gefasst und genutzt werden kann. Im Sommer kann auch in Zukunft noch ähnlich viel Wasser den Gewässern entnommen werden, da die Zuflussmengen in den meisten Fällen immer noch grösser als die Fassungskapazitäten sind.

Der bei der Fallstudie KW Prättigau gefundene Effekt der besseren Ausnützung der Fassungskapazitäten im Winter und damit verbunden die Erhöhung der Stromproduktion in der Periode 2021-2050 - wird durch die andern im Rahmen von CCWasserkraft durchgeführten Fallstudien bestätigt: Für den Sommer zeigen die Ergebnisse keine Veränderung oder aber eine Abnahme der Stromproduktion. Über das ganze Jahr gesehen sind insgesamt nur kleine Veränderungen zu erwarten; vor allem im südlichen und östlichen Wallis liegen sie eher im leicht negativen Bereich, wobei mit Unsicherheiten von $\pm 10 \%$ gerechnet werden muss (SGHL und CHy, 2011).

Aussagen für die ferne Zukunft (2070-2099) sind noch sehr unsicher; sie hängen von der regional unterschiedlichen Entwicklung der Niederschläge sowie der Höhenlage und dem Vergletscherungsgrad der Einzugsgebiete ab. Aufgrund der bisherigen Erkenntnisse sind die Trends in der Stromproduktion vor allem für die hoch gelegenen, heute noch stark vergletscherten Gebiete des südlichen und östlichen Wallis sowie für die Alpensüdseite negativ.

\subsection{Auswirkungen der Klimaänderung auf die Stromproduktion in der gesamten Schweiz}

Bei den Hochrechnungen der oben dargestellten Ergebnisse auf die Schweiz wurde von zwei Varianten ausgegangen: Der optimistische Fall geht von der Annahme aus, dass selbst bei projizierten Abflussabnahmen im Sommer die Fassungskapazitäten von Laufkraftwerken nicht unterschritten werden, und diese somit keine Produktionseinbussen haben. Im pessimistischen Fall hingegen wirken sich tiefere Abflussmengen direkt auf die Produktion von Laufkraftwerken aus, womit diese weniger produzieren. In Tabelle 3 sind die Ergebnisse zusammengestellt. Bei beiden Varianten ergibt sich für die Schweiz eine leicht positive mittlere Produktionserwartung. Unter Berücksichtigung der Unsicherheiten, die insgesamt nur grob zu quantifizieren sind, kann man das Ergebnis der Hochrechnung so interpretieren, dass sich über das Jahr gesehen gegenüber heute keine signifikanten Veränderungen in der mittleren Stromproduktionserwartung ergeben werden.

Bemerkenswert sind allerdings die Veränderungen im Sommer- und Winterhalbjahr: Die Stromproduktion im Sommer wird in der Schweiz insgesamt, aber auch bei den meisten Kraftwerksgruppen - hier mehrheitlich im Bereich von $-3 \%$ bis $-10 \%$ - abnehmen. Für den Winter hingegen wird bei den meisten Kraftwerken eine Zunahme der Stromproduktion zwischen $+2 \%$ und $+26 \%$ erwartet. Einzig bei Kraftwerken im Südtessin muss auch im Winter mit Abnahmen gerechnet werden. Auf die Schweiz hochgerechnet ergibt sich für die nahe Zukunft eine projizierte Zunahme der Energieproduktion im Winter um $+10.1 \%$. Ökonomisch gesehen wird davon ausgegangen, dass die winterliche Produktionssteigerung die sommerlichen Verluste zu kompensieren vermag, da typischerweise unter den gegeben Marktbedingungen die Grosshandelspreise in der Schweiz im Winter höher liegen als im Sommer.

Für die fernere Zukunft, also für die Periode 2070-2099, gelten diese Aussagen nicht: Modellrechnungen zeigen beispielsweise, dass die Abflussmengen aus vergletscherten Gebieten signifikant abnehmen werden (Farinotti et al., 2011), einhergehend mit einem allmählichen Verschwinden der Gletscher bis Ende des Jahrhunderts (Paul et al., 2011) und einer Abnahme der sommerlichen Niederschlagsmengen (Bosshard et al., 2011).

\section{Fazit}

Wie in der Einleitung dargestellt, wurden die Auswirkungen der Klimaänderung auf die Wasserkraftnutzung bereits 2007 abgeschätzt (BFE, 2007). Man ging von einer klimabedingten Abnahme der mittleren Stromproduktion von $7 \%$ 
aus. Diese Zahl basiert dabei auf einer Untersuchung, in welcher die Veränderungen der natürlichen Abflussmengen in elf alpinen Einzugsgebieten für die Periode 2020-2049 aufgrund von älteren, weniger hoch aufgelösten Klimaszenarien (Christensen et al., 2002) abgeschätzt wurden (Horton et al., 2005). Die Resultate der hier vorgestellten Fallstudien zeigen nun aber, dass eine Hochrechnung auf die Schweiz basierend allein auf Projektionen natürlicher Abflussmengen problematisch ist, da sich eine Veränderung der natürlich anfallenden Wassermenge nicht zwingend linear auf die Stromproduktion übertragen lässt. Die im Rahmen von CCWasserkraft durchgeführte Hochrechnung auf die Schweiz ging deshalb einen wesentlichen Schritt weiter; sie berücksichtigte neben den Veränderungen in den natürlichen Abflussmengen auch die technische Auslegung der Wasserkraftanlagen.

Gestützt werden die Ergebnisse der schweizerischen Studie CCWasserkraft durch eine vergleichbare Untersuchung für Österreich (Schöner et al., 2011), die von einer mittleren jährliche Zunahme der Stromproduktion um +0.5 bis $+2.5 \%$ ausgeht (2021-2050 im Vergleich zu 1976-2006).

Insgesamt sind für 2021-2050 zwar Veränderungen in der saisonalen mittleren Stromproduktion zu erwarten; die Jahresproduktion wird sich aber wenig verändern. Was heisst das nun für die Betreiber von Wasserkraftanlagen? Nach Spreng $(2011,312)$,dürfte die klimabedingte Betriebsoptimierung keine grossen Probleme mit sich bringen." Veränderungen auf Seiten des Strommarktes sowie die wetterbedingte Variabilität von Jahr zu Jahr seien grösser als die durch die Klimaänderung verursachten Trends. „Die Betriebsoptimierung muss allen Veränderungen kurzfristig gerecht werden und wird dabei die steten, aber von Moment zu Moment und Jahr zu Jahr kaum wahrnehmbaren klimabedingten Veränderungen auffangen." Paul Michellod, Direktor der Walliser Kraftwerke FHV, wies in einem Vortrag in Visp (September 2011) aber auch auf Probleme hin, die auf die Kraftwerksbetreiber zukommen könnten. Er betonte insbesondere zwei Faktoren:

1. Durch eine mögliche Zunahme von grossen Hochwasserereignissen könnte sich das kurzfristige Wasserdargebot erhöhen.

2. Durch den Rückgang der Gletscher werden im hochalpinen Bereich vermehrt Geröllflächen freigelegt und damit das Angebot an Sediment massiv erhöht.

Die Kombination dieser beiden Faktoren werde, so Michellod, zu einer vermehrten Versandung von Wasserfassungen und Staubecken führen und hätte damit negative Auswirkungen auf die Stromproduktion. Neben solchen Risiken könnten durch die Klimaänderung aber auch neue Chancen entstehen. So könnten nach Haeberli et al. (2012) nach einem Gletscherrückgang neue Seen entstehen, welche beispielsweise von der Wasserkraft als Speicher, Sedimentrückhalt oder zum Hochwasserschutz genutzt werden könnten. Das zuvor genannte Beispiel aus der Praxis belegt, dass die Auswirkungen der Klimaänderung auf die Was- serkraftnutzung im Einzelfall je nach geographischer Lage, Einzugsgebiet und Anlagetyp beträchtlich sein können, und dies trotz insgesamt günstigeren Prognosen für die mittlere Stromproduktion der Schweiz in naher Zukunft.

Edited by: M. Hoelzle

Reviewed by: three anonymous referees

\section{Literatur}

AF-Colenco AG: WABES, ein Programmsystem zur wasserwirtschaftlichen Betriebssimulation von Kraftwerksstufen, Eigenentwicklung, Baden, AF-Colenco AG, 2004.

BAFU: Auswirkungen der Klimaänderung auf Wasserressourcen und Gewässer. Synthesebericht zum Projekt "Klimaänderung und Hydrologie in der Schweiz" (CCHydro), Bundesamt für Umwelt, Bern, Umwelt-Wissen Nr. 1217, 76 S., 2012.

Balmer, M.: Nachhaltigkeitsbezogene Typologisierung der schweizerischen Wasserkraftanlagen. GIS-basierte Clusteranalyse und Anwendung in einem Erfahrungskurvenmodell, Dissertation der ETH Zürich, Zürich, 2011.

BFE: Die Energieperspektiven 2035. - Band 1 Synthese Modellrechnungen, Vergleiche, Bewertungen und Herausforderungen, Bern, BFE, 2007.

BFE: Bundesrat konkretisiert Stossrichtung der Energiestrategie 2050, Medienmitteilung vom 25. Mai 2012, Bern, BFE, 2011.

Blöchlinger, L., Bollinger, T., Maurer, J., und Semadeni, M.: An evaluation model for the market-to-market value of hydropower plants, Proceedings of the 6th IAEE European Conference "Modelling in Energy Economics and Policy", Zürich, ETH Zürich, 2004.

Bosshard, T.: Hydrological climate-impact modeling in the Rhine catchment down to Cologne, Diss. ETH 19861, Zürich, 1-140, 2011.

Bosshard, T., Kotlarski, S., Ewen, T., und Schär, C.: Spectral representation of the annual cycle in the climate change signal, Hydrol. Earth Syst. Sci., 15, 2777-2788, doi:10.5194/hess-152777-2011, 2011.

CH2011: Swiss Climate Change Scenarios CH2011, Zürich: C2SM, MeteoSwiss, ETH, NCCR Climate and OcCC, 2011.

Christensen, J. H., Carter, T., und Giorgi, F.: PRUDENCE employs new methods to assess European climate change, EOS 82/13, 147, 2002.

Fachberichte: Fachberichte zum Projekt Auswirkungen der Klimaänderung auf die Wasserkraftnutzung, http://www.hydrologie. unibe.ch/projekte/ccwasserkraft.html (letzter Zugriff: 20 July 2012), 2011.

Farinotti, D., Bauder, A., und Funk, M.: Veränderung der Gletscher und ihrer Abflüsse 1900-2100. Fallstudien Gornergletscher und Mattmark, Wasser-Energie-Luft, 103, 273-278, 2011.

Haeberli, W., Schleiss, A., Linsbauer, A., Künzler, M., und Bütler, M.: Gletscherschwund und neue Seen in den Schweizer Alpen Perspektiven und Optionen im Bereich Naturgefahren und Wasserkraft, Wasser-Energie-Luft, 104, 93-102, 2012.

Hänggi, P.: Auswirkungen der hydroklimatischen Variabilität auf die Wasserkraftnutzung in der Schweiz. Inauguraldissertation der Philosophischen-naturwissenschaftlichen Fakultät der Universität Bern, Bern, 1-206, 2011. 
Hänggi, P. und Weingartner, R.: Interannual variability of runoff and climate within the Upper Rhine River basin 1808-2007, Journal of Hydrological Sciences, 56, 34-50, 2011.

Hänggi, P., Angehrn, S., Bosshard, T., Helland, E., Job, D., Rietmann, D., Schädler, B., Schneider, R., und Weingartner, R.: Einfluss der Klimaänderung auf die Stromproduktion der Wasserkraftwerke Löntsch und Prättigau, Wasser-Energie-Luft, 103, 292-299, 2011a.

Hänggi, P., Bosshard, T., und Weingartner, R.: Swiss discharge regimes in a changing climate, in: Hänggi, P.: Auswirkungen der hydroklimatischen Variabilität auf die Wasserkraftnutzung in der Schweiz, Inauguraldissertation der Philosophischnaturwissenschaftlichen Fakultät der Universität Bern, Bern, 77100, $2011 b$.

Hänggi, P., Balmer, M., und Weingartner, R.: Auswirkung der Klimaänderung auf die Wasserkraftnutzung in der Schweiz - Hochrechnung, Wasser-Energie-Luft, 103, 300-307, 2011c.

Horton, P., Schaefli, B., Mezghani, A., Hingray, B., und Musy, A.: Prediction of climate change impacts on Alpine discharge regimes under A2 and B2 SRES emission scenarios for two future time periods, Energiewirtschaftliche Grundlagen, Bern, Bundesamt für Energie, 2005.

Huss, M., Farinotti, D., Bauder, A., und Funk, M.: Modelling runoff from highly glacierized alpine drainage basins in a changing climate, Hydrol. Process., 22, 3888-3902, 2008.

IPCC: Climate Change 2007. Synthesis Report. Contribution of Working Groups I, II and III to the Fourth Assessment Report of the Intergovernmental Panel on Climate Change, Geneva, IPCC, 2007.

Magnusson, J., Farinotti, D., Jonas, T., und Bavay, M.: Quantitative evaluation of different hydrological modeling approaches in a partly glacierized Swiss watershed, Hydrol. Process., 25, 20712084, 2011.

MeteoSchweiz: Klima Schweiz - Trends heute, http: //www.meteoschweiz.admin.ch/web/de/klima/klima_heute/ trends_schweiz.html (letzter Zugriff: März 2013), 2012.

Paul, F., Linsbauer, A., und Haeberli, W.: Grossräumige Modellierung von Schwundszenarien für alle Schweizer Gletscher. Fachbericht für die Synthese des Projektes Klimaänderung und Wasserkraftnutzung, Zürich: Geographisches Institut, Universität Zürich, 2011.
Raymond-Pralong, M., Turowski, J., Rickenmann, D., Beer, A., Métraux, V., und Glassey, T.: Auswirkungen der Klimaänderung auf die Geschiebefracht in Einzugsgebieten von Kraftwerksanlagen im Kanton Wallis, Wasser-Energie-Luft, 103, 278-285, 2011.

Schöner, W., Böhm, R., Haslinger, K., Blöschl, G., Merz, R., Blaschke, A. P., Viglione, A., J. Parajka, Kroiß, H., Kreuzinger, N., und Hörhan, Th.: Anpassungsstrategien an den Klimawandel für Österreichs Wasserwirtschaft, Wien, Bundesministerium für Land- und Forstwirtschaft, Umwelt und Wasserwirtschaft, 2011.

SGHL und CHy: Auswirkungen der Klimaänderung auf die Wasserkraftnutzung. Synthesebericht, Beiträge zur Hydrologie der Schweiz 18, Bern, 1-28, 2011.

Spreng, D.: Klimawandel: Handlungsbedarf für die schweizerischen Wasserkraftbetreiber?, Wasser-Energie-Luft, 103, 308312, 2011.

Stähli, M., Raymond-Pralong, M., Zappa, M., Ludwig, A., Paul, F., Bosshard, Th., und Dupraz, Ch.: Klimaänderung und Wasserkraft. Fallstudie Kraftwerke Oberhasli AG. Fachbericht für die Synthese des Projektes Klimaänderung und Wasserkraftnutzung, Birmensdorf, Eidg. Forschungsanstalt für Wald, Schnee und Landschaft, 2011.

Van der Linden, P. und Mitchell, J. F. B. (Hrsg.): ENSEMBLES: Climate Change and its Impacts. Summary of research and results from the ENSEMBLES project, Exeter, UK, Met Office Hadley Center, 2009.

Viviroli, D., Zappa, M., Gurtz, J., und Weingartner, R.: An introduction to the hydrological modelling system PREVAH and its preand post-processing tools, Environ. Modell. Softw., 24, 12091222, 2009.

Weingartner, R. und Aschwanden, H.: Abflussregimes als Grundlage zur Abschätzung von Mittelwerten des Abflusses, Hydrologischer Atlas der Schweiz, Bern, Tafel 5.2., 1992. 